\title{
Elevated Urinary Matrix Metalloproteinase-7 Detects Underlying Renal Allograft Inflammation and Injury
}

\author{
Julie Ho, MD, ${ }^{1,2,3}$ David N. Rush, MD, ${ }^{1}$ Oleg Krokhin, PhD, ${ }^{2}$ Mihaela Antonovici, MSc, ${ }^{4}$ Ang Gao, ${ }^{2}$ \\ Jennifer Bestland, ${ }^{5}$ Chris Wiebe, MD, ${ }^{1}$ Brett Hiebert, MD, ${ }^{6}$ Claudio Rigatto, MD, ${ }^{1}$ Ian W. Gibson, MD, ${ }^{7}$ \\ John A. Wilkins, PhD, ${ }^{2}$ and Peter W. Nickerson, $\mathrm{MD}^{1,2,3}$
}

Background. The urinary CXC chemokine ligand (CXCL)10 detects renal transplant inflammation noninvasively, but has limited sensitivity and specificity. In this study, we performed urinary proteomic analysis to identify novel biomarkers that may improve the diagnostic performance of urinary CXCL10 for detecting alloimmune inflammation in renal transplant patients. Methods. In preliminary studies, adult renal transplant patients with normal histology $(n=5)$, interstitial fibrosis and tubular atrophy $(n=6)$, subclinical $(n=6)$ and clinical rejection $(n=6)$, underwent in-depth urine protein compositional analysis with LC-MS/MS, and matrix metalloproteinase-7 (MMP7) were identified as a potential candidate for the diagnosis of renal allograft inflammation. Urine MMP7 performance was then studied in a larger, prospective adult renal transplant population $(n=148$ urines from $n=133$ patients) with matched surveillance/indication biopsies. The diagnostic performance of urinary MMP7 and CXCL10 in combination was next evaluated using concordance (C-) statistics, net reclassification improvement and integrated discrimination improvement indices, to determine whether it was better than CXCL10 alone. Results. Urinary MMP7:creatinine $(\mathrm{Cr})$ was lower in normal transplants compared to those with inflammation: glomerulonephritis $(P=0.009)$, viral nephropathies $(P=0.002)$, interstitial fibrosis and tubular atrophy and inflammation $(P=0.04)$, borderline $(P=0.08)$, subclinical $(P=0.01)$ and clinical rejection $(P=0.0006)$, and acute tubular necrosis $(P<0.0001)$. Urinary MMP7:Cr and CXCL10:Cr significantly distinguished noninflamed from inflamed biopsies (area under the curve, 0.74 and 0.70, respectively). The addition of urinary MMP7:Cr to CXCL10:Cr improved the diagnostic performance for subclinical and clinical inflammation/injury by integrated discrimination improvement $(P=0.002)$ and net reclassification improvement $(P=0.006)$ analyses. Conclusions. Urinary MMP7:Cr improves the overall diagnostic performance of urinary CXCL10:Cr for distinguishing normal histology from subclinical and clinical inflammation/injury, but not subclinical inflammation alone.

(Transplantation 2016;100: 648-654)

Oriog ne of the major challenges of transplantation is the optimization of immunosuppressive therapy to balance the risk of rejection from underimmunosuppression against the risk of infection/malignancy from overimmunosuppression. ${ }^{1}$

Received 3 October 2014. Revision requested 27 April 2015.

Accepted 4 May 2015.

${ }^{1}$ Section of Nephrology, Department of Internal Medicine, University of Manitoba, Winnipeg, Manitoba, Canada.

${ }^{2}$ Manitoba Centre for Proteomics and Systems Biology, University of Manitoba and Health Sciences Centre, Winnipeg, Manitoba, Canada.

${ }^{3}$ Department of Immunology, University of Manitoba, Winnipeg, Manitoba, Canada.

${ }^{4}$ Institute for Technical Biochemistry, University of Stuttgart, Stuttgart, Germany.

${ }^{5}$ University of Manitoba, Winnipeg, Manitoba, Canada.

${ }^{6}$ Cardiac Sciences Program, Winnipeg Regional Health Authority, Winnipeg, Manitoba, Canada.

${ }^{7}$ Department of Pathology, University of Manitoba, Winnipeg, Manitoba, Canada. This study was funded by the Canadian Institutes of Health Research (CIHR) and the Norman S Coplon Satellite Healthcare Extramural Grant program. J.H. has salary support from the Manitoba Medical Services Foundation Dr. F.W. Du Val Clinical Research Professorship. PN holds the Flynn Family Chair in Renal Transplantation, University of Manitoba.

The authors declare no conflicts of interest.

J.H. designed the study, attained the operating funds, supervised the experimental work and analysis, and wrote the article. D.R. contributed to collection of the clinical
The ideal regimen would provide the minimum immunosuppression required to prevent subclinical and clinical rejection while limiting infections that have a negative impact on graft survival (eg, polyoma virus). Developing tailored

database/urine bio-bank and writing the article. O.K. supervised the mass spectrometry based proteomic analysis and writing the article. M.A. performed the mass spectrometry based proteomic analysis and assisted with writing the mass spectrometry methods in the article. A.G. performed all the urinary MMP7 enzymelinked immunosorbent assay assays. C.W. assisted with the statistical analysis and contributed to writing the article. B.H. performed the statistical analysis and contributed to writing the article. C.R. supervised the statistical analysis and assisted with the article regarding the statistical methodology section. I.G. evaluated the histopathology and contributed to writing the article. J.A.W. supervised the mass spectrometry based proteomic analysis and writing the article. P.N. contributed to collection of the clinical database/urine bio-bank. He also contributed to the study design, data analysis and writing the article.

Correspondence: Julie Ho, MD, FRCPC, Sections of Nephrology and Biomedical Proteomics, University of Manitoba, GE421C, Health Sciences Center, 820 Sherbrook Street, Winnipeg MB R3A 1R9. (jho@hsc.mb.ca).

Supplemental digital content (SDC) is available for this article. Direct URL citations appear in the printed text, and links to the digital files are provided in the HTML text of this article on the journal's Web site (www.transplantjournal.com).

Copyright @ 2015 Wolters Kluwer Health, Inc. All rights reserved.

ISSN: 0041-1337/16/10003-648

DOI: 10.1097/TP.0000000000000867 
immunosuppression regimens require sensitive, noninvasive tools for serial monitoring after drug minimization/ withdrawal protocols to detect subclinical inflammation before injury, and also to follow the response to antirejection treatment.

Multiple noninvasive biomarkers have been evaluated for the detection of acute cellular rejection. ${ }^{2}$ Several groups have demonstrated that the urinary chemokines $\mathrm{CXC}$ chemokine ligand (CXCL) 9 and CXCL10 are sensitive markers for inflammation and are associated with acute rejection. ${ }^{3-12}$ Furthermore, urinary CXCL9 and CXCL10 levels increase before the rise in serum creatinine $(\mathrm{Cr})^{3,10,11}$ and decrease after treatment of rejection. ${ }^{3,9-11}$ Finally, early elevations in urinary CXCL10 and CXCL9 have been associated with decreased renal allograft function at 6 and 24 months, respectively. ${ }^{3,10}$

Although urinary CXCL9, CXCL10, granzyme A, and perforin can detect subclinical inflammation, ${ }^{5-7,10,12}$ only urinary CXCL10 has had its diagnostic performance for subclinical inflammation validated in independent cohorts. ${ }^{5-7}$ However, urinary CXCL10 achieved only modest discrimination for subclinical rejection (area under the curve [AUC], 0.69 ), suggesting that a panel of markers may be needed. ${ }^{5}$ Therefore, the goal of this study was to use in-depth mass spectrometry analysis to identify novel biomarkers that might improve the overall diagnostic performance of urinary CXCL10.

\section{MATERIALS AND METHODS}

\section{Discovery Proteomics Cohort}

All analyses were conducted with informed consent and the protocol approved by the University of Manitoba Health Research Ethics Board. Urinary proteomics was performed in a small, highly selected cohort of adult renal transplant patients with matched biopsy and clinical data $(\mathrm{n}=23$ urines from 14 patients). Maintenance immunosuppression consisted of mycophenolate mofetil, prednisone, and a calcineurin inhibitor (cyclosporine, $\mathrm{n}=7$; tacrolimus, $\mathrm{n}=7$ ). Urines were collected before surveillance or indication biopsies and frozen at $-80^{\circ} \mathrm{C}$ for analysis. Multiple samples were evaluated from individuals to control for inter-individual variability. Biopsies were scored according to the Banff 2007 criteria. ${ }^{13}$ The groups were:

(1) Normal renal transplant function and histology $(n=5)$ :

(a) Acute: $\mathrm{i} 0 \mathrm{t} 0 \mathrm{v} 0 \mathrm{~g} 0$

(b) Chronic: ci 0 ct 0 cv0 cg0

(2) Interstitial fibrosis and tubular atrophy (IFTA, $n=6$ ):

(a) Acute: $\mathrm{i}+\mathrm{t} \leq 1 \mathrm{v} 0 \mathrm{~g} 0$

(b) Chronic: ci $\geq 1 \mathrm{ct} \geq 1 \pm \mathrm{cv}>1 \mathrm{cg} 0$

(3) Subclinical rejection $(\mathrm{n}=6)$ :

(a) Acute: $\mathrm{i} \geq 2 \mathrm{t} \geq 2 \mathrm{v} 0 \mathrm{~g} 0$

(b) Chronic: ci0 ct0 $\pm \mathrm{cv}>1 \mathrm{cg} 0$

(4) Clinical rejection $(n=6)$ :

(a) Acute: $\mathrm{i} \geq 2 \mathrm{t} \geq 2 \mathrm{v} 0 \mathrm{~g} 0$

(b) Chronic: $\mathrm{ci}+\mathrm{ct} \leq 1 \pm \mathrm{cv}>1 \mathrm{cg} 0$

\section{Quantitative Test Cohort}

A candidate biomarker identified from the in-depth LC-MS/MS analysis was quantitatively studied in a larger, selected adult renal transplant population. ${ }^{7}$ In brief, this population consisted of 148 urines from 133 patients with matched surveillance or indication biopsies. Maintenance immunosuppression was predominantly mycophenolate mofetil, prednisone, and tacrolimus; and high immunological risk patients received thymoglobulin induction therapy. The groups were:

(1) Normal transplant function and histology $(\mathrm{n}=22)$ :

(a) Acute: i0-1 t0 g0 v0

(b) Chronic: ci + ct $\leq 1 \mathrm{cv} 0 \mathrm{cg} 0$

(2) Glomerulonephritis (GN) ( $\mathrm{n}=15)$

(a) Recurrent IgA nephropathy, $\mathrm{n}=11$

(b) Recurrent focal segmental glomerulosclerosis, $\mathrm{n}=3$

(c) De novo membranous, $\mathrm{n}=1$

(3) $\operatorname{Viral}(\mathrm{n}=7)$

(a) Polyoma nephropathy with polyoma virus viremia, $\mathrm{n}=6$

(b) Adenovirus nephropathy ${ }^{14} \mathrm{n}=1$

(4) IFTA $(\mathrm{n}=20)$ :

(a) Acute: i0-1 t0 g0 v0

(b) Chronic: $\mathrm{ci} \geq 1 \mathrm{ct} \geq 1 \pm \mathrm{cv}>1 \pm \mathrm{cg}>1$

(5) IFTA and inflammation $(\mathrm{n}=13)$ :

(a) Acute: i1-2 t1 g0 v0

(b) Chronic: $\mathrm{ci} \geq \mathrm{ct} \geq 1 \pm \mathrm{cv}>1 \pm \mathrm{cg}>1$

(6) Borderline tubulitis $(\mathrm{n}=13)$ :

(a) Acute: i1-2 t1 g0 v0

(7) Subclinical rejection $(\mathrm{n}=17)$ :

(a) Acute: $\mathrm{i} \geq 2 \mathrm{t} \geq 2$

(b) Serum $\mathrm{Cr}<20 \%$ from baseline.

(c) All subclinical tubulitis biopsies were surveillance biopsies.

(8) Clinical T cell mediated rejection (TCMR) $(\mathrm{n}=17)$ :

(a) Acute: $\mathrm{i} \geq 2 \mathrm{t} \geq 2$

(b) Serum $\mathrm{Cr} \geq 20 \%$ from baseline.

(c) All clinical tubulitis biopsies were indication biopsies.

(9) Antibody mediated rejection (AMR) $(\mathrm{n}=10)$ :

(a) Donor specific antibody positive.

(b) C4d positive, with peritubular capillaritis.

(10) Acute tubular necrosis (ATN) $(\mathrm{n}=14)$

(a)Serum $\mathrm{Cr} \geq 20 \%$ from baseline.

\section{Proteomics Analysis}

Monoclonal antibodies were produced against purified human serum albumin, protein A, apolipoprotein, B2microglobulin, haptoglobin, and human $\operatorname{IgG}$, and validated 
by enzyme-linked immunosorbent assay (ELISA), Western blot, or mass spectrometry (data not shown). A depletion column was prepared with these antibodies to deplete urine samples $(3 \mathrm{~mL})$ of high abundance proteins. Urines were then concentrated, reduced, alkylated, digested, and analyzed on 2-dimensional LC-MS/MS as previously described ${ }^{15}$ (details in the Supplemental Methods, SDC, http://links.lww.com/TP/B179). Proteins that were common and unique to each histopathological group were identified and the lists annotated using PubMed and Uniprot. Matrix metalloproteinase-7 (MMP7) was identified as a potential biomarker candidate for further evaluation.

\section{ELISA Analysis}

Urinary CXCL10 was quantified by ELISA; the sensitivity was $1.95 \mathrm{pg} / \mathrm{mL}$, and the intra-assay and interassay coefficients of variation were 3.90 and $4.54 \%$, respectively. ${ }^{5-7}$ Urinary MMP7 was quantified by ELISA (R\&D Systems, Catalog DY907). The sensitivity was $7.8 \mathrm{pg} / \mathrm{mL}$, and the intra-assay and interassay coefficients of variation were $2.71 \%$ and $6.15 \%$, respectively. The ELISAs were read on a Biotek Synergy 4 microplate reader (Gen 5 software, Fisher Scientific). Urine Cr was measured with a Cobas analyser (Roche Diagnostics) and used to correct for dilutional factors.

\section{Statistical Analysis}

Statistical analysis was performed using SAS Institute version 9.3, Copyright 2012 SAS Institute Incorporated, Cary NC software. Results are presented as means \pm SD for continuous variables. Frequencies of categorical variables are presented as counts and percentages. Mann-Whitney $U$ tests were used for nonparametric data. Urinary MMP7:Cr and CXCL10:Cr were analyzed in both native units and log transformed variables. Univariate logistic regression was performed and a receiver operating characteristic AUC was generated using urinary MMP7:Cr and CXCL10:Cr as continuous variables. A range of sensitivities and specificities were generated for urinary MMP7:Cr based on different AUC cutoff values.

Using the methods of Pencina et al, ${ }^{16}$ the following were calculated: the increment in the $\mathrm{C}$ statistic occurring with addition of urinary MMP7:Cr to CXCL10:Cr; the net reclassification improvement (NRI) of the enriched versus base model; and the integrated discrimination improvement (IDI) of the enriched model versus base model. We determined that urinary MMP7:Cr would be a clinically useful test if its addition to CXCL10:Cr resulted in statistically and clinically meaningful improvements in all 3 measures, defined as an increase in C-statistic greater than 0.05 , NRI greater than $10 \%$, and relative IDI greater than $10 \%$.

\section{RESULTS}

\section{Discovery Proteomics}

Protein compositional analysis was performed on 23 urines from 14 patients and their demographics are detailed in Table S1 (SDC, http://links.lww.com/TP/B179). As expected, renal function was better in the normal and subclinical rejection groups as compared to the IFTA and clinical rejection groups. Proteinuria was not different between groups. The subclinical and clinical rejection biopsies occurred earlier after transplantation than IFTA biopsies, but this did not reach statistical significance likely due to the small numbers.

The number of proteins identified in individual urines ranged from 157 to 617 (Table S2, SDC, http://links.lww. com/TP/B179). Eighteen proteins were common to the subclinical and clinical rejection groups, whereas 137 and 77 proteins were unique to each group, respectively. The MMP7 (Uniprot P09237) was identified as a candidate (clinical rejection $n=4$, subclinical rejection $n=1$, IFTA $n=1$ and normal transplant $\mathrm{n}=1$ ) to be evaluated in the quantitative test cohort, whose patient and urine/biopsy characteristics are described in Tables $1 \mathrm{~A}$ and $1 \mathrm{~B}$.

\section{Urinary MMP7:Cr Identifies Inflamed/Injured Renal Allografts}

Urinary MMP7:Cr (ng/mmol) was low in noninflamed allografts with normal histology and IFTA, respectively $(264 \pm 168,469 \pm 678, P=0.41)$. Conversely, urinary MMP7:Cr was significantly elevated with GN (616 \pm 450 , $P=0.009)$; viral nephropathies $(1033 \pm 1063, P=0.002)$; IFTA + inflammation $(471 \pm 350, P=0.04)$, subclinical $(735 \pm 693, P=0.01)$ and clinical rejection $(906 \pm 896$, $P=0.0006)$, and ATN $(14300 \pm 39695, P<0.0001)$ compared to normal. Urinary MMP7:Cr also showed a trend to significance in borderline inflammation $(468 \pm 387)$ compared to normal renal allografts $(P=0.08)$. Importantly, urinary MMP7:Cr was significantly elevated in subclinical rejection (Fig. 1A).

As expected, urinary CXCL10:Cr was elevated in viral nephropathies, borderline, subclinical, and clinical $\mathrm{TCMR}^{5,7}$ (Fig. 1B). Interestingly, urinary MMP7:Cr distinguished underlying GN from normal histology $(P=0.009)$, whereas CXCL10:Cr did not. The AMR demonstrated low urinary CXCL10:Cr as previously reported, ${ }^{5}$ and this was mirrored by low MMP7:Cr levels (Fig. 1). We postulate that urinary biomarkers only detect inflammation/injury in the tubulointerstitial compartment, whereas microvascular injury may be better identified with serum biomarkers. The AMR was therefore censored from the diagnostic performance analysis.

\section{Urinary MMP7:Cr and CXCL10:Cr Individual Diagnostic Performance}

Urinary MMP7:Cr distinguished noninflamed (normal transplant and IFTA) from inflamed/injured allografts (GN, viral nephropathies, IFTA + inflammation, borderline,

\section{TABLE 1A.}

Patient characteristics, $n=133$

\begin{tabular}{lc}
\hline Patient characteristics & $\mathbf{n}$ \\
\hline Recipient age, y & $46 \pm 12$ \\
Sex, male & $88(66)$ \\
Race, white & $91(68)$ \\
Delayed graft function, dialysis within the first & $17 / 131(13)$ \\
$\quad$ week after transplantation & \\
Maintenance immunosuppression, mycophenolate & $97(73)$ \\
$\quad$ mofetil/tacrolimus/prednisone & \\
Donor, living & $62(47)$ \\
Donor age, y & $39 \pm 14$ \\
HLA mismatch & $3.2 \pm 1.4$ \\
\hline
\end{tabular}

Reported as mean \pm SD, or count (\%). 
subclinical and clinical rejection, and ATN) with an AUC of 0.74 (Table 2A), whereas urinary CXCL10:Cr had an AUC of 0.70 (Table 2B). The diagnostic performance of urinary MMP7:Cr and CXCL10:Cr were both evaluated for detecting subclinical pathologies, which resulted in an AUC of 0.61 and 0.74 , respectively (Table 2A-B). The sensitivities and specificities of urinary MMP7:Cr at different cutoff values is reported (Table S3, SDC, http:/links.lww.com/TP/B179).

\section{Urinary MMP7:Cr and CXCL10:Cr Combined Diagnostic Performance}

The addition of urinary MMP7:Cr to CXCL10:Cr improved the overall diagnostic performance for distinguishing noninflamed from inflamed/injured allografts (AUC, 0.76 [95\% confidence interval, $0.67-0.84])$ by IDI $(P=0.002)$ and NRI $(P=0.006)$ analyses (Table $2 \mathrm{~B}-\mathrm{C})$. This was consistent with an overall C-statistic improvement of 0.06 , which met our prespecified criteria for clinical significance (C-statistic $>0.05$ ); however, this did not reach statistical significance $(95 \%$ confidence interval, -0.01 to $0.12, P=0.10$ ). Urinary MMP7:Cr did not improve the overall diagnostic performance of CXCL10:Cr for detecting subclinical pathologies (Table 2B-C).

\section{DISCUSSION}

In this study, unbiased proteomic analyses identified urinary MMP7 as a potential candidate for the detection of renal allograft inflammation/injury; and indeed urinary MMP7 was found to noninvasively detect renal allograft inflammation/injury and outperform serum $\mathrm{Cr}$ by identifying subclinical rejection. As there is evidence that MMP7 and CXCL10 exert their effects via different pathophysiological pathways, we hypothesized that their combination would improve on the diagnostic performance afforded by CXCL10 alone. The major finding of this study is the combination of urinary MMP7 and CXCL10 significantly improved the overall diagnostic performance for distinguishing noninflamed from inflamed/injured renal allografts. However, the addition of urinary MMP7 did not improve on the ability to detect subclinical inflammation of urinary CXCL10 alone.

Different unbiased approaches have previously identified MMPs as potential rejection biomarkers. Ling et $\mathrm{al}^{17}$ performed a mass spectrometry-based peptidomic analysis and identified a 40-peptide and 6-gene urine biomarker panel for rejection, which included MMP7. They did not evaluate subclinical rejection and could not determine if their 40peptide panel outperformed serum $\mathrm{Cr} .{ }^{17}$ Metzger et a ${ }^{18}{ }^{18}$ identified collagen fragments in subclinical and clinical rejection urines with a peptidomic analysis. These collagen $\alpha(\mathrm{I})$ and $\alpha$ (III) fragments were thought to be cleavage products of MMP8, but they only identified MMP8 in tissue and not urine ${ }^{18}$ Furthermore, mass spectrometry-based peptidomics is difficult to translate to routine clinical platforms compared to ELISA. Rodder et $\mathrm{al}^{19,20}$ performed microarray analyses on acute rejection and IFTA biopsies, and determined that MMP7 was significantly enriched in acute rejection, ${ }^{19}$ but tissue arrays cannot be used for noninvasive surveillance. ${ }^{19}$ Finally, Hirt-Minkowski et $\mathrm{al}^{21}$ demonstrated that urinary MMP7 was significantly associated with subclinical tubulitis. Taken together, our study independently confirms these findings and further extends them by evaluating their clinical 
A

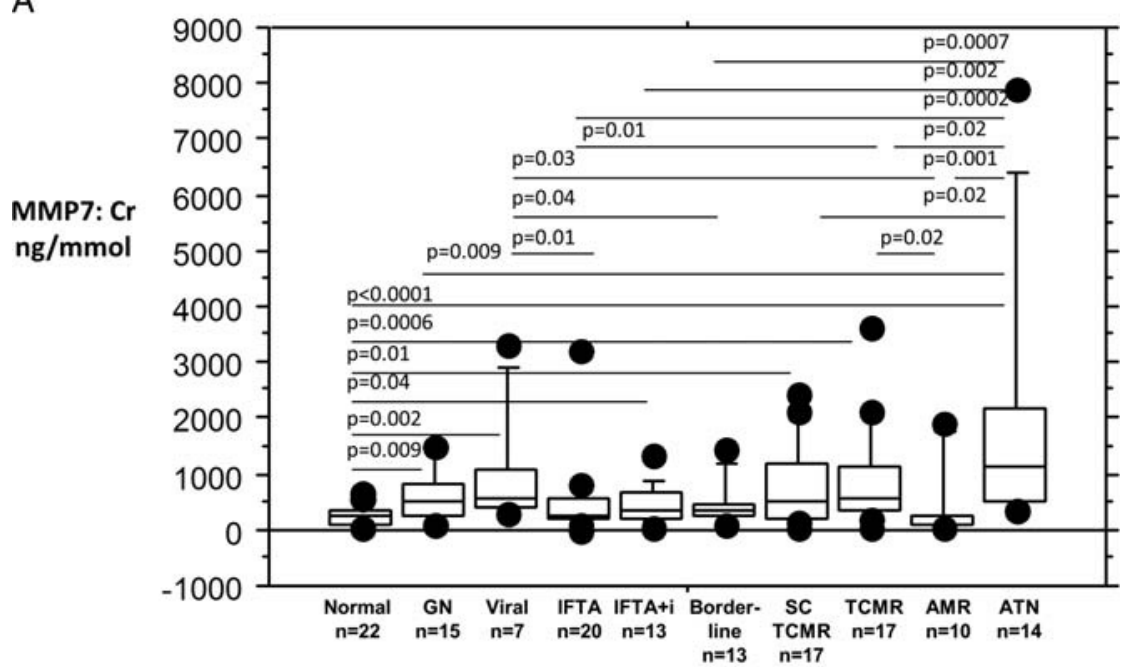

B

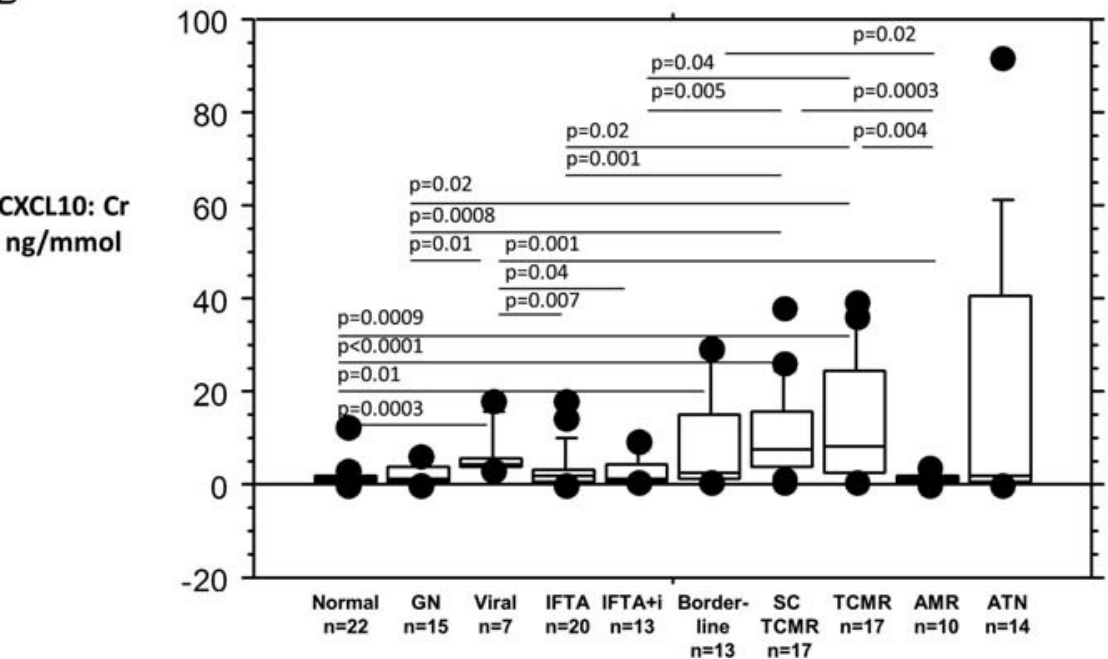

FIGURE 1. Urinary MMP7:Cr and CXCL10:Cr distinguish healthy from inflamed/injured renal allografts. IFTA + i indicates IFTA with inflammation; SC TCMR, subclinical TCMR.

utility in combination with CXCL10 and with additional diagnostic categories.

MMP7 is a downstream effector of the canonical Wnt signalling pathway and its expression is regulated by $\beta$-Catenin; it has pleiotropic functions, including the degradation of extracellular matrix proteins. The MMP7 is not detectable in normal renal biopsies, ${ }^{22}$ but localizes to the apical region of renal tubular epithelial cells in chronic kidney disease,

\section{TABLE 2A.}

Urinary MMP7:Cr distinguishes normal histology from inflamed renal transplant parenchyma

\begin{tabular}{|c|c|c|c|c|}
\hline Group & Model & C-Statistic (95\% CI) & Odds Ratio (95\% Cl) & $\boldsymbol{P}$ \\
\hline \multirow{6}{*}{$\begin{array}{l}\text { Normal/IFTA versus IFTA and } \\
\text { inflammation/borderline/subclinical/clinical/GN/viral/ATN }\end{array}$} & MMP7 ( $\uparrow 100)$ & $0.74(0.65-0.83)$ & $1.19(1.06-1.34)$ & 0.005 \\
\hline & MMP7 $\geq 200$ & $0.60(0.52-0.68)$ & $3.46(1.43-8.36)$ & 0.006 \\
\hline & MMP7 $\geq 400$ & $0.67(0.59-0.75)$ & $4.23(1.90-9.42)$ & $<0.001$ \\
\hline & MMP7 $\geq 600$ & $0.66(0.59-0.72)$ & $6.62(2.18-20.04)$ & $<0.001$ \\
\hline & MMP7 $\geq 800$ & $0.63(0.58-0.70)$ & $9.69(2.20-42.70)$ & 0.003 \\
\hline & MMP7 $\geq 1000$ & $0.62(0.57-0.68)$ & $15.45(2.02-118.14)$ & 0.008 \\
\hline \multirow[t]{6}{*}{ Normal/IFTA versus IFTA and inflammation/borderline/subclinical } & MMP7 $(\uparrow 100)$ & $0.66(0.54-0.78)$ & $1.11(0.99-1.24)$ & 0.08 \\
\hline & MMP7 $\geq 200$ & $0.56(0.47-0.66)$ & $1.89(0.71-5.01)$ & 0.20 \\
\hline & MMP7 $\geq 400$ & $0.61(0.51-0.71)$ & 2.69 (1.08-6.69) & 0.03 \\
\hline & MMP7 $\geq 600$ & $0.60(0.52-0.69)$ & 4.12 (1.22-13.92) & 0.02 \\
\hline & MMP7 $\geq 800$ & $0.57(0.50-0.64)$ & 4.57 (0.91-22.97) & 0.07 \\
\hline & MMP7 $\geq 1000$ & $0.58(0.52-0.64)$ & 9.37 (1.12-78.64) & 0.04 \\
\hline
\end{tabular}


TABLE 2B.

C-Statistic comparisons between models with CXCL10 + MMP7 compared to CXCL10 only model

\begin{tabular}{|c|c|c|c|c|c|}
\hline Group & Model & $\begin{array}{c}\text { CXCL10 Only } \\
\text { C-Statistic } \\
\text { (95\% CI) }\end{array}$ & $\begin{array}{l}\text { Model C-Statistic } \\
\quad(95 \% \mathrm{CI})\end{array}$ & $\begin{array}{c}\text { C-Statistic } \\
\text { Improvement } \\
(95 \% \mathrm{CI})\end{array}$ & $P$ \\
\hline Normal/IFTA versus IFTA and & CXCL10 + MMP7 & $0.70(0.61-0.79)$ & $0.76(0.67-0.84)$ & $0.06(-0.01$ to 0.12$)$ & 0.10 \\
\hline inflammation/borderline/subclinical/clinical/GN/viral/ATN & CXCL10 + MMP7 $\geq 1000$ & $0.70(0.61-0.79)$ & $0.73(0.64-0.81)$ & $0.02(-0.01$ to 0.05$)$ & 0.11 \\
\hline Normal/IFTA versus IFTA and & CXCL10 + MMP7 & $0.74(0.63-0.84)$ & $0.74(0.63-0.84)$ & $0.001(-0.04$ to 0.04$)$ & 0.96 \\
\hline inflammation/borderline/subclinical & CXCL10 + MMP7 $\geq 1000$ & $0.74(0.63-0.84)$ & $0.75(0.64-0.85)$ & $0.01(-0.02$ to 0.04$)$ & 0.44 \\
\hline
\end{tabular}

consistent with tubular secretion. ${ }^{22,23}$ Conversely, CXCL10 is secreted by infiltrating inflammatory cells, renal tubular and mesangial cells. ${ }^{24,25}$ CXCL10 acts as a chemotactic agent for leukocyte recruitment in renal allograft rejection and helps mediate the Th1 helper response. ${ }^{24,25}$ Notably, $\beta$-Catenin signalling appears to be independent of chemokine induction. In a mouse model of cellular inflammation using Citrobacter rodentium infection, $\beta$-Catenin inhibition had no impact on interferon- $\gamma$, CXCL10 and CXCL9 induction. $^{26}$ Conversely, there is no literature to suggest that CXCR3 chemokines can activate Wnt/ $\beta$-Catenin. We demonstrated that MMP7 combined to improve the overall diagnostic performance of urinary CXCL10 alone for subclinical/ clinical injury, and we postulate that this is because they act via different pathophysiological pathways. However, this is strictly an observational study in which association can be determined but not causation.

Although the role of MMP7 in renal allograft rejection remains uncharacterized, it is known to be elevated in response to injury. In a mouse model of obstructive uropathy and focal segmental glomerulosclerosis, He et $\mathrm{al}^{21}$ demonstrated that activation of $\mathrm{Wnt} / \beta$-Catenin signalling resulted in increased renal MMP7 expression which closely correlated with urinary MMP7 excretion. Interestingly, MMP7 induced E-cadherin degradation in a time- and dose-dependent manner in proximal tubular epithelial cells, suggesting that it may be upregulated to help mediate renal tubular epithelial cell repair. ${ }^{23}$

The development of novel biomarkers has been broadly categorized into the following phases: discovery, performance evaluation, and impact determination ${ }^{27}$; and this last validation step has been performed by few transplant biomarker studies to date. ${ }^{4,28}$ An important strength of this study is that it spanned biomarker discovery to impact determination and had multiple levels of validation to carefully characterize the clinical utility of urinary MMP7. First, the mass spectrometry results were confirmed with ELISA, demonstrating a significant association of MMP7 with inflammation/ injury. Second, evaluation of subclinical rejection demonstrated that urinary MMP7 outperforms the clinical standard, serum Cr. Third, urinary MMP7 was evaluated against and in combination with another biomarker with demonstrated ability to detect subclinical alloimmune inflammation: urinary CXCL10. Finally, these results were also externally consistent with the published literature.

There are some limitations to this study. First, the clinicalpathological classification of patients in both cohorts was dependent on allograft histology, which may be subject to sampling error. Second, this is a retrospective analysis and there is insufficient data on peritubular capillaritis (Banff ptc score), peritubular capillary C4d deposition, and donor-specific antibody status, to reclassify all patients according to the updated Banff 2013 schema. ${ }^{29}$ Therefore, it is possible that mixed rejection phenotypes may contaminate the cellular rejection groups. Nevertheless, the observed low urinary CXCL10 levels in AMR are externally consistent with the reported literature. ${ }^{5}$ These low CXCL10 and MMP7 levels suggest that if the TCMR group is contaminated with mixed rejection, the overall effect would be to dilute the findings and not inflate them. Finally, paricalcitol inhibits both renal MMP7 expression and urinary MMP7 excretion in a mouse model of adriamycin nephropathy. ${ }^{17}$ Our group consistently uses calcitriol, and not paricalcitol, for the treatment of secondary hyperparathyroidism. However, it is possible that calcitriol may have a similar inhibitory effect on MMP7 expression and excretion, and this was not controlled for in this study.

In conclusion, urinary MMP7 is elevated in renal allograft inflammation/injury and has moderate discrimination for subclinical rejection. MMP7 elevation likely reflects activation of the Wnt/ $\beta$-catenin signalling pathway in response to injury. Urinary MMP7 in combination with CXCL10 improves the overall diagnostic performance when considering subclinical and clinical pathologies, likely indicating that its role in these processes is different from that of CXCL10. Thus, MMP7 may provide additional insights regarding the pathologic processes involved in renal injury associated with allograft inflammation.

TABLE 2C.

IDI and NRI between models with CXCL10 + MMP7 compared to CXCL10 only model

\begin{tabular}{|c|c|c|c|c|c|}
\hline Group & Model & IDI (95\% CI) & IDI, $P$ & NRI (95\% CI) & NRI, $P$ \\
\hline Normal/IFTA versus IFTA and & CXCL10 + MMP7 & $0.05(0.02-0.08)$ & 0.0016 & $0.51(0.17-2.77)$ & 0.006 \\
\hline inflammation/borderline/subclinical/clinical/GN/viral/ATN & CXCL10 + MMP7 $\geq 1000$ & $0.03(0.01-0.06)$ & 0.0062 & $0.48(0.10-2.58)$ & 0.01 \\
\hline Normal/IFTA versus IFTA and & CXCL10 + MMP7 & 0.007 (-0.01 to 0.03$)$ & 0.47 & $0.13(-0.26$ to 0.51$)$ & 0.56 \\
\hline inflammation/borderline/subclinical & CXCL10 + MMP7 $\geq 1000$ & $0.02(-0.01$ to 0.06$)$ & 0.17 & $0.32(0.07-0.58)$ & 0.13 \\
\hline
\end{tabular}




\section{ACKNOWLEDGMENTS}

The authors thank Evelyn Roloff for her assistance in preparation of the article. The authors also thank Dr. David Lovett for his helpful input.

\section{REFERENCES}

1. Kirk AD, Mannon RB, Swanson SJ, et al. Strategies for minimizing immunosuppression in kidney transplantation. Transp/ Int. 2005; 18:2-14.

2. Ho J, Wiebe C, Gibson IW, et al. Immune monitoring of kidney allografts. Am J Kidney Dis. 2012;60:629-640.

3. Hricik DE, Nickerson P, Formica RN, et al. Multicenter validation of urinary CXCL9 as a risk-stratifying biomarker for kidney transplant injury. Am J Transplant. 2013;13:2634-2644.

4. Tatapudi RR, Muthukumar T, Dadhania D, et al. Noninvasive detection of renal allograft inflammation by measurements of mRNA for IP-10 and CXCR3 in urine. Kidney Int. 2004;65:2390-2397.

5. Hirt-Minkowski P, Amico P, Ho J, et al. Detection of clinical and subclinical tubulo-interstitial inflammation by the urinary CXCL10 chemokine in a real life setting. Am J Transplant. 2012;12:1811-1823.

6. Schaub S, Nickerson P, Rush D, et al. Urinary CXCL9 and CXCL10 levels correlate with the extent of subclinical tubulitis. Am J Transplant. 2009;9: 1347-1353.

7. Ho J, Rush DN, Karpinski M, et al. Validation of urinary CXCL10 as a marker of borderline, subclinical and clinical tubulitis. Transplantation. 2011;92:878-882.

8. $\mathrm{Hu} \mathrm{H}, \mathrm{Kwun} \mathrm{J}$, Aizenstein BD, et al. Noninvasive detection of acute and chronic injuries in human renal transplant by elevation of multiple cytokines/ chemokines in urine. Transplantation. 2009;87:1814-1820.

9. Hu H, Aizenstein BD, Puchalski A, et al. Elevation of CXCR3-binding chemokines in urine indicates acute renal-allograft dysfunction. Am J Transplant. 2004;4:432-437.

10. Matz M, Beyer J, Wunsch D, et al. Early post-transplant urinary IP-10 expression after kidney transplantation is predictive of short- and long-term graft function. Kidney Int. 2006;69:1683-1690.

11. Hauser IA, Spiegler S, Kiss E, et al. Prediction of acute renal allograft rejection by urinary monokine induced by IFN-y (MIG). J Am Soc Nephrol. 2005;16:1849-1858.

12. Jackson JA, Kim EJ, Begley B, et al. Urinary chemokines CXCL9 and CXCL10 are noninvasive markers of renal allograft rejection and BK viral infection. Am J Transplant. 2011;11:2228-2234.

13. Solez K, Colvin RB, Racusen LC, et al. Banff 07 classification of renal allograft pathology: updates and future directions. Am J Transplant. 2008;8: 753-760.

14. Storsley L, Gibson IW. Adenovirus interstitial nephritis and rejection in an allograft. J Am Soc Nephrol. 2011;22:1423-1427.
15. Dwivedi RC, Spicer V, Harder M, et al. Practical implementation of $2 \mathrm{D}$ HPLC scheme with accurate peptide retention prediction in both dimensions for high-throughput bottom-up proteomics. Anal Chem. 2008;80: 7036-7042.

16. Pencina MJ, D'Agostino RB Sr, D'Agostino RB Jr, et al. Evaluating the added predictive ability of a new marker: from area under the ROC curve to reclassification and beyond. Stat Med. 2008;27:157-172.

17. Ling XB, Sigdel TK, Lau K, et al. Integrative urinary peptidomics in renal transplantation identifies biomarkers for acute rejection. J Am Soc Nephrol. 2010;21:646-653.

18. Metzger J, Chatzikyrkou C, Broecker V, et al. Diagnosis of subclinical and clinical acute T-cell mediated rejection in renal transplant patients by urinary proteome analysis. Proteomics Clin Appl. 2011;5: 322-333.

19. Rodder S, Scherer A, Korner M, et al. Meta-analyses qualify metzincins and related genes as acute rejection markers in renal transplant patients. Am J Transplant. 2010;10:286-297.

20. Rodder S, Scherer A, Raulf F, et al. Renal allografts with IF/TA display distinct expression profiles of metzincins and related genes. Am J Transplant. 2009;9:517-526.

21. Hirt-Minkowski P, Marti H-P, Honger G, et al. Correlation of serum and urinary matrix metalloproteases/tissue inhibitors of metalloproteases with subclinical allograft fibrosis in renal transplantation. Transp/ Immunol. 2014;30:1-6.

22. Surendran K, Simon TC, Liapis H, et al. Matrilysin (MMP-7) expression in renal tubular damage: association with Wnt4. Kidney Int. 2004;65: 2212-2222.

23. He W, Tan RJ, Li Y, et al. Matrix metalloproteinase-7 as a surrogate marker predicts renal Wnt/ß-Catenin activity in CKD. J Am Soc Nephrol. 2012;23:294-304.

24. el-Sawy T, Fahmy NM, Fairchild RL. Chemokines: directing leukocyte infiltration into allografts. Curr Opin Immunol. 2002;14:562-568.

25. Dufour JH, Dziejman M, Liu MT, et al. IFN-gamma-inducible protein 10 (IP10; CXCL10)-deficient mice reveal a role for IP-10 in effector T cell generation and trafficking. J Immunol. 2002;168:3195-3204.

26. Brown JB, Cheresh P, Goretsky T, et al. Epithelial phosphatidylinositol3-kinase signaling is required for $\beta$-Catenin activation and host defense against Citrobacter rodentium infection. Infect Immun. 2011; 79:1863-1872.

27. Parikh CR, Thiessen-Philbrook H. Key concepts and limitations of statistical methods for evaluating biomarkers of kidney disease. J Am Soc Nephrol. 2014;25:1621-1629.

28. Suthanthiran M, Schwartz JE, Ding R, et al. Urinary-cell mRNA profile and acute cellular rejection in kidney allografts. N Engl J Med. 2013; 369:20-31.

29. Haas M, Sis B, Racusen LC, et al. Banff 2013 meeting report: inclusion of C4d-negative antibody-mediated rejection and antibody-associated arterial lesions. Am J Transplant. 2014;14:272-283. 